\title{
Coexistence of ferromagnetism and singlet superconductivity via kinetic exchange
}

\author{
Mario Cuoco, ${ }^{1,2}$ Paola Gentile, ${ }^{1}$ and Canio Noce ${ }^{1,3}$ \\ ${ }^{1}$ Unità I.N.F.M. di Salerno, Dipartimento di Fisica "E. R. Caianiello", \\ Università di Salerno, I-84081 Baronissi (Salerno), Italy \\ ${ }^{2}$ Centre de Recherches sur les Très Basses Températures associé à l'Université Joseph Fourier, \\ C.N.R.S., BP 166, 38042 Grenoble-Cédex 9, France \\ ${ }^{3}$ Unità I.N.F.M. di Salerno-Coherentia, Salerno, Italy
}

\begin{abstract}
We propose a novel mechanism for the coexistence of metallic ferromagnetism and singlet superconductivity assuming that the magnetic instability is due to kinetic exchange. Within this scenario, the unpaired electrons which contribute to the magnetization have a positive feedback on the gain of the kinetic energy in the coexisting phase by undressing the effective mass of the carriers involved into the pairing. The evolution of the magnetization and pairing amplitude, and the phase diagram are first analyzed for a generic kinetic exchange model and then are determined within a specific case with spin dependent bond-charge occupation.
\end{abstract}

The problem of the interplay between ferromagnetic (FM) and superconducting (SC) long range order has been recently attracting new interest due to the discovery of superconductivity in ferromagnetic metals, $\mathrm{UGe}_{2}$ [1], $\mathrm{ZrZn}_{2}$ [2], URhGe [3], and in rutheno-cuprate $\mathrm{RuSr}_{2} \mathrm{RECu}_{2} \mathrm{O}_{8}$ compounds, with $\mathrm{RE}=\mathrm{Eu}$ or $\mathrm{Gd}[4]$.

The investigation of ferromagnetic superconductors started by analyzing the case of two interacting subsystems: one formed by localized spins or aligned magnetic impurities which produces the ferromagnetic background, the other composed by itinerant electrons which gives rise to the superconductivity. Within this framework, early works [5, 6, 7] focused on singlet superconductivity in presence of a spin-exchange field, showing that it can exist only below a critical value of the magnetic coupling. Hence, with the purpose to increase the threshold of the critical spin-exchange, it was suggested a finite momentum pairing state coexisting with the ferromagnetic order [8, 9]. In this pairing configuration, commonly indicated as the Fulde-Ferrell-Larkin-Ovchinnikov state, the subtle balance between the condensate energy of the Cooper pairs with a finite center-of-mass momentum and the Zeeman energy, related to the magnetic moments of depaired itinerant electrons, was able to account for a superconducting-ferromagnetic (SF) phase in presence of spin-exchange higher than the zero-momentum pairing state.

Nevertheless, in the above mentioned materials, a new phenomenology seems to arise if compared to the conventional case of a metal with magnetic impurities. Indeed, it has been suggested that (i) ferromagnetism and superconductivity are cooperative phenomena, (ii) the FM state is due to itinerant electrons, (iii) the same electrons participate in both the FM and SC order, and (iv) for the case of systems with two types of carrier responsible for the SC and FM phases separately, there are interesting cooperative effects due to competing charge- and spinexchange coupling. Theoretical studies along those directions have been recently performed by considering the occurrence of ferromagnetism and superconductivity in ei- ther singlet 10, 11, 12, 13] and/or triplet 14, 15, 16] channel of pairing. In particular, as far as the SC state with singlet $s$-wave pairing is concerned, it has been shown 17 . that its coexistence with weak itinerant ferromagnetism can be obtained within a single band model, where the ferromagnetic order is driven by the same electrons that participate in the formation of Cooper pairs. One crucial aspect of such analysis concerns the stability of the $\mathrm{SF}$ state. The loss of condensation energy due to the depaired electrons which produce a non zero total magnetization, is not compensated by the energy gain of the magnetic exchange, thus the SF state turns out always to be energetically unfavorable against the non-magnetic SC one even if $s$ - and $d$-wave symmetry or finite-momentum pairing state are considered [18].

In this letter, we propose a novel mechanism for the coexistence of superconductivity and ferromagnetism within a single band model. The new and crucial ingredient is that the metallic ferromagnetism is not due, as in the previous studies, to a rigid shift in the positions of the majority and minority spin bands (i.e. Stoner model), but it is a consequence of a change in the relative bandwidth of electrons with up and down spin polarization. In this circumstance, the gain of energy comes from the undressing of the mass for the majority spins which induces a bandwidth enlargement and in turn lowers the kinetic energy 19. When the pairing interaction is switched on, the interplay between the gain in kinetic energy, due to the relative change of the majority and minority spin bands, and the condensation energy which would tend to pair all the electrons becomes crucial for the stability of the SF state. It turns out that such a phase is the most favorable only if a suitable tuning of the ratio between the density of depaired and paired states is reached, so to optimize the balance in the kinetic and potential energy gain. We will analyze this possibility in two steps: I) the case in which the variation between the mass of up and down spin electrons is arbitrary modified without referring to any microscopic mechanism is firstly considered; II) a specific tight-binding model in which off-diagonal 
Coulomb interactions are responsible for an asymmetric bond-charge distribution for each spin channel, is then investigated. For both cases, the attractive interaction will be assumed of BCS-type.

Model I. Let us start from a model Hamiltonian which contains a local attractive potential and itinerant electrons with a spin dependent mass:

$$
\begin{aligned}
H_{I}= & -t \sum_{\langle i j, \sigma\rangle}\left(2 w_{\sigma}\right)\left(c_{i \sigma}^{\dagger} c_{j \sigma}+H . c .\right)-g \sum_{i} c_{i \uparrow}^{\dagger} c_{i \downarrow}^{\dagger} c_{i \downarrow} c_{i \uparrow} \\
& -\mu \sum_{i \sigma} c_{i \sigma}^{\dagger} c_{i \sigma}
\end{aligned}
$$

where $c_{i \sigma}^{\dagger}\left(c_{i \sigma}\right)$ creates (destroys) an electron with spin $\sigma$ at the site $i, w_{\sigma}$ is a positive term that controls the renormalization of the mass for electrons with spin $\sigma$ (the factor 2 being introduced for convenience), $\mu$ is the chemical potential, $t$ is the hopping amplitude which defines the bare bandwidth, and $g$ is the pairing coupling being effective only in a shell of amplitude $2 \omega_{c}$ around the Fermi surface as in the usual BCS theory. It is worth pointing out that in this case the mass dressing and undressing can be generated both via the coupling to a background of spin, as in double-exchange systems, or to dynamical processes intrinsically generated by electron correlations.

The introduction of a pairing amplitude, after the mean-field decoupling of the attractive term, brings to the following diagonal expression for the Hamiltonian:

$$
\begin{aligned}
H_{I-M F} & =\sum_{k}\left(E_{k}^{\alpha} \alpha_{k}^{\dagger} \alpha_{k}+E_{k}^{\beta} \beta_{k}^{\dagger} \beta_{k}\right)+E_{0} \\
E_{0} & =\sum_{k}\left[-E_{k}^{\beta}+\left(2 w_{\downarrow} \epsilon_{k}-\mu\right)\right]+\frac{\Delta^{2}}{g},
\end{aligned}
$$

where $\epsilon_{k}=-t \sum_{\delta} \exp (i k \cdot \delta)$ is the bare dispersion with $\delta$ being a vector connecting a site to its nearest neighbors, and $\Delta=\sum_{k} g\left\langle c_{k \uparrow} c_{-k \downarrow}\right\rangle$ is the pairing amplitude, respectively. The field operators $\alpha_{k}, \beta_{k}$ correspond to fermionic excitations with quasiparticle dispersion

$$
E_{k}^{\alpha, \beta}= \pm a \epsilon_{k}+\sqrt{\left(b \epsilon_{k}-\mu\right)^{2}+\Delta^{2}} .
$$

$b=w_{\uparrow}+w_{\downarrow}$ and $a=w_{\uparrow}-w_{\downarrow}$ being the average and half the difference of the spin mass renormalization. It is immediately apparent that the kinetic exchange amplitude is proportional to $a$ while the value of $b$ determines the order of magnitude of the average kinetic energy. Still, the sign of the total magnetization $(M)$ follows that of $a$, while $b$ is always positive. Thus, without any loss of generality one can focus only on the case with $a \geq 0$, the other one being just derived by inverting the direction of $M$.

The equations for the pairing amplitude and the mag- netization have the following form

$$
\begin{aligned}
\Delta & =\frac{g \Delta}{2} \sum_{k} \frac{1-n_{k}^{\alpha}-n_{k}^{\beta}}{\sqrt{\left(b \epsilon_{k}-\mu\right)^{2}+\Delta^{2}}} \\
M & =\frac{\mu_{B}}{2} \sum_{k}\left(n_{k}^{\beta}-n_{k}^{\alpha}\right),
\end{aligned}
$$

where $n_{k}^{\alpha, \beta}$ are the Fermi distributions in the momentum space of the fermionic fields having $E_{k}^{\alpha, \beta}$ as energy dispersion relations, respectively, and $\mu_{B}$ is the Bohr magneton. Let us now discuss under what conditions the system can accommodate a coexistence of SC and FM order. The analysis will be restricted to the zero temperature limit and furthermore we will assume that the density of electrons $n$ is self-consistently fixed by a positive value of the chemical potential $\mu$. The results can be symmetrically extended within the same procedure to the negative $\mu$ case. Hence, considering that $b, a, \Delta, \mu \geq 0$ and $b \geq a$, it is possible to show that $n_{k}^{\alpha}=0$ as $E_{k}^{\alpha}$ is always positive, while $n_{k}^{\beta}=1$ when $\lambda_{k,-} \leq \epsilon_{k} \leq \lambda_{k,+}$, where $\lambda_{k, \pm}=\frac{b \mu \pm \sqrt{a^{2}\left(\Delta^{2}+\mu^{2}\right)-b^{2} \Delta^{2}}}{b^{2}-a^{2}}$. The $\lambda_{k, \pm}$ define a range on the positive side $\epsilon_{k}$, which is different from zero only when $a$ and $b$ are linked to the amplitude of $\Delta$ and $\mu$ in a way to fulfill the relation $\frac{a}{b}>\frac{\Delta}{\sqrt{\Delta^{2}+\mu^{2}}}$. If the previous inequality holds, single particle states form within the gap and contribute to give a finite magnetization. Then, depending on their density the system can allow for a state with coexisting SC and FM order. In the limit of $\mu \rightarrow 0(n \sim 1)$ or $a \rightarrow 0(M \sim 0)$, there are no real solutions for $\lambda_{k, \pm}$ so that one ends up with the usual BCS-like state with zero magnetization. In the other cases, the self-consistent equations for the gap amplitude and the number of electrons have to be solved to determine the conditions for the existence of SF state. For such purpose, it has been used a model density of states given by $N(\epsilon)=1 / 2 w$ (with $w=2 t$ ) if $-w \leq \epsilon \leq w$. We notice that, contrary to the case of the Stoner model, a non-trivial ferromagnetic solution can be got without the need of a curvature in the density of states close to the Fermi level [19]. The analysis of the solutions is performed by fixing the value of $b$ and $n$ and studying how the pairing amplitude, the energy and the magnetization are modified by changes of $a$. Due to the formation of unpaired electrons within the gap, the value of the effective average mass in the SF state $\left(b_{s f}\right)$ is modified with respect to the case of zero magnetization $\left(b_{s c}\right)$. Hence, there might occur two distinct cases depending on the microscopic mechanism which controls the relative change of the majority and minority spin bandwidth: 1) undressing of the average effective mass, i.e. $\delta b>0 ; 2)$ dressing of the average effective mass, i.e. $\delta b<0, \delta b \equiv\left(b_{s f}-b_{s c}\right) / b_{s c}$ being the relative percentage variation.

In Fig 1 is shown the comparison between the energy of the SF and the SC state, together with the behav- 
ior of the magnetization and the pairing amplitude as a function of the relative shift between the mass of up and down spin electrons. For simplicity, we have fixed $b_{s c}=1$ and we have studied how the difference of energy is modified in the cases (1) and (2). Changes in $b_{s c}$ produce only quantitative but not qualitative differences in the results. As shown in the top panel of Fig 1 only when $\delta b$ is positive and for a suitable density of the depaired states, controlled by the amplitude of $a$, the system can be stabilized in a SF phase. When the average mass increases, though the effective amplitude pairing grows and in turn lowers the condensation energy, the loss in the kinetic energy of the electrons that participate in the pairing cannot be counterbalanced to get a stable SF state. It is worth pointing out that the transition from the SF state to the $\mathrm{SC}$ one is of first order for the magnetization but it is continuous for the pairing amplitude by moving from small to large values of $a$, while it is reversed in the opposite $a$ direction. Nevertheless, depending on the amplitude of the undressing $\delta b$, the transition from the SF state can be first order type in both the order parameters. Finally, due to the peculiar link between $\Delta$ and $\mu$, we notice that the change in the density of electrons modifies the region where the SF solution exists. In particular, the interval of solutions shrinks if one moves towards the half-filling case, whereas a large band undressing is required to stabilize the SF phase.

Model II. Now we consider an explicit tight-binding model where the bandwidth change depends on the bondcharge occupation in each spin channel[19]. The model Hamiltonian has the following form:

$$
\begin{aligned}
& H_{I I}=-t \sum_{<i j>\sigma}\left(c_{i \sigma}^{\dagger} c_{j \sigma}+H . c\right)+U \sum_{i} n_{i \uparrow} n_{i \downarrow} \\
& +V \sum_{<i j>} n_{i} n_{j}+J \sum_{<i j>\sigma \sigma^{\prime}}\left(c_{i \sigma}^{\dagger} c_{j \sigma^{\prime}}^{\dagger} c_{i \sigma^{\prime}} c_{j \sigma}+H . c\right) \\
& +J^{\prime} \sum_{<i j>}\left(c_{i \uparrow}^{\dagger} c_{i \downarrow}^{\dagger} c_{j \downarrow} c_{j \uparrow}+H . c\right)-g \sum_{i}\left(c_{i \uparrow}^{\dagger} c_{i \downarrow}^{\dagger} c_{i \downarrow} c_{i \uparrow}+H . c\right) \\
& -\mu \sum_{i \sigma} c_{i \sigma}^{\dagger} c_{i \sigma}
\end{aligned}
$$

where $U$ and $V$ represent the on-site and nearestneighbor Coulomb repulsion, respectively, and the parameters $J$ and $J^{\prime}$ describe nearest-neighbor exchange and pair hopping processes, while $g$ is the BCS pairing. By applying the Hartree-Fock decoupling, one can obtain the expression of the bare quasi-particle dispersion in terms of a bond charge quantity $I_{\sigma}=\left\langle c_{i \sigma}^{\dagger} c_{j \sigma}\right\rangle$ that in the momentum space is given by $I_{\sigma}=\sum_{k} n_{k \sigma}\left(\frac{-\epsilon_{k}}{w}\right)$ in a way that the kinetic part of the Hamiltonian (77) (neglecting the Stoner exchange) reads $H_{I I-M F}=$ $\sum_{k \sigma}\left[\left(1-2 j_{1} I_{\sigma}-2 j_{2} I_{-\sigma}\right) \epsilon_{k}-\mu\right] n_{k \sigma}$. It is possible to show that the values of $j_{1}$ and $j_{2}$ are linked to the $V, J$, and $J^{\prime}$ interactions by means of the following relations: $j_{1}=(J-V) / w$ and $j_{2}=\left(J+J^{\prime}\right) / w[19]$. Hence, by

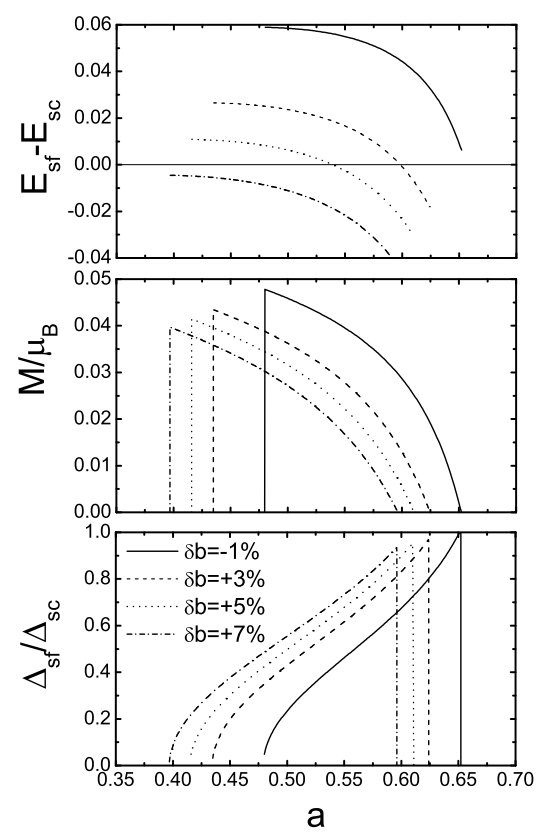

FIG. 1: From the bottom to the top: superconducting gap, total magnetization, and relative energy between the SF and the $\mathrm{SC}$ phase, respectively, as a function of $a$, for different values of the average effective mass renormalization. The density of electrons has been fixed to $n=1.2$ and the coupling strength is $g / t=1$.

following the same procedure as in Model I, it is convenient to introduce the parameters $a$ and $b$, which now has to be determined self-consistently via the following relations: $b=\left[1-\left(j_{1}+j_{2}\right)\left(I_{\uparrow}+I_{\downarrow}\right)\right], a=\left(j_{2}-j_{1}\right)\left(I_{\uparrow}-I_{\downarrow}\right)$ Still, $I_{\sigma}$ depends on $a$ and $b$ by means of the occupation number of the $\alpha(\beta)$ bands, via the following relations:

$$
\begin{aligned}
& n_{k \uparrow}=v_{k}^{2}+u_{k}^{2}\left(n_{k}^{\alpha}+n_{k}^{\beta}\right)-n_{k}^{\beta} \\
& n_{k \downarrow}=v_{k}^{2}+u_{k}^{2}\left(n_{k}^{\alpha}+n_{k}^{\beta}\right)-n_{k}^{\alpha} .
\end{aligned}
$$

where $u_{k}^{2}=\left(\frac{1}{2}\right)\left(1+\frac{\left(b \epsilon_{k}-\mu\right)}{\sqrt{\left(b \epsilon_{k}-\mu\right)^{2}+\Delta^{2}}}\right)$ and $v_{k}^{2}=1-u_{k}^{2}$ are the coefficients of the Bogoliubov transformation.

Few comments are worth mentioning on the possible solutions and on the effect of depaired electrons in the amplitude of the average mass $b$ within the SF state. First of all, the amplitude of $a$ and $b$ is determined by $j_{-}=\left(j_{2}-j_{1}\right)$ and $j_{+}=\left(j_{1}+j_{2}\right)$, respectively. Moreover, the sign of $j_{+}$controls whether the starting value in SC state for $b$ is larger or smaller than 1 , while $j_{-}$sets the sign of the magnetization. As far as the stability is concerned, like in the Model I, it is crucial to see whether the introduction of depaired electrons in the SF phase reduces or increases the average effective mass with respect to that of the SC state. Hereafter, we will assume that $j_{ \pm}$are positive, which is consistent, in the weak coupling regime, with realistic values of the microscopic 
couplings $\left(J, J^{\prime}, V\right)$ above introduced.

Let us now analyze the dependence of $b$ in the case of the SF and SC phase. One can show that $b_{s f}$ gets undressed in the coexisting phase, thus its value is larger than $b_{s c}$ when $n_{k}^{\beta} \neq 0$. This result can be deduced by writing down the explicit expression of the ground state energy of the SF and SC phases. The expectation value of the bond charge term on the unpaired electrons gives a negative contribution to $I_{\uparrow}+I_{\downarrow}$ which is not present in the non-magnetic SC case, thus increasing the average kinetic energy and consequently the value of $E_{0}$ in the coexisting state.

In Fig. 22 it is reported the phase diagram for $j_{+}=0.5$, $g / t=0.25$ obtained by varying $j_{-}$with respect to the density. Of course, by modifying the amplitude of $j_{+}$one can span the phase diagram for all the possible values of $j_{1}$ and $j_{2}$. We have checked that a change in $j_{+}$yields a shift in the critical line but does not give any qualitative change in the phase diagram. The phenomenology of the transition between the SC and SF state is the same of that observed for the Model I. Indeed, when one goes through the critical line, the magnetization has a jump to its possible maximal value which depends on $\Delta$ and $\mu$, while the SC gap grows continuously from zero. For completeness, in Fig 2] it is also reported the line of transition from a paramagnetic to a FM state in the case of absence of superconductivity. The shape of the region of stability in the $\left[\left(j_{-}-j_{+}\right), n\right]$ diagram can be understood by noticing that the kinetic exchange is given by $k_{e x}=\epsilon_{\uparrow}-\epsilon_{\downarrow}=2 a \epsilon$ and $a=2 j_{-} m(n-1)$ (for the pure ferromagnetic case). Thus approaching the half-filling limit $(n \rightarrow 1)$ one has that $a \rightarrow 0$ and consequently the kinetic exchange goes to zero so that it is needed an infinite value of $j_{-}$to get a spin polarized state. This consideration explains the asymptotic behaviour of the critical line as we get close to the half-filling limit where it becomes more and more difficult to have a magnetized state because the difference in the bond charge occupation for opposite spin becomes close to zero thus requiring high values of the coupling constants to create a charge unbalance.

In conclusion, we have studied the occurrence of ferromagnetism and $s$-wave singlet superconductivity within a single band model where the magnetic moments are due to a kinetic exchange mechanism, both for a generic and for a specific case with bond-charge coupling. It has been shown that the depaired electrons play a crucial role in the energy balance and that only when their dynamical effect is such to undress the effective mass of the carriers which participate in the pairing, then the SF phase can be stabilized. As far as the specific case of a bond-charge kinetic mechanism is concerned, we have shown that the phase diagram has a peculiar dependence on the density of carriers, and that only when the system is far from the limit of exact particle-hole symmetry, the SF phase can be obtained without going to very large couplings.

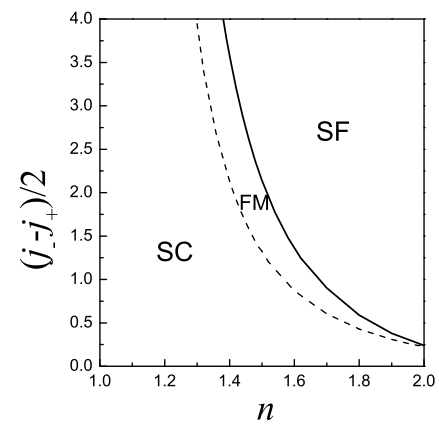

FIG. 2: Phase diagram relative to the Model II, showing the transition(full line) from the SF to the SC state, and that one from a paramagnetic to FM state when $\Delta=0$ (dashed line), as the difference $\left(j_{-}-j_{+}\right)$and the density of electrons are varied. Due to the particle-hole symmetry, the part for $n$ in the range $[0,1]$ is just symmetrically related.

M.C. acknowledges support from the European program "Improving Human Potential". The authors thank Maria Teresa Mercaldo for helpful comments and valuable discussions.

[1] S. S. Saxena et al., Nature (London) 406, 587 (2000).

[2] C. Pfeiderer et al., Nature (London) 412, 58 (2001).

[3] D. Aoki et al., Nature (London) 413, 613 (2001).

[4] J. Tallon et al., IEEE Trans. Appl. Supercon. 9, 1696 (1999); C. Bernhard et al., Phys. Rev B 59, 14099 (1999).

[5] A. A. Abrikosov and L. P. Gorkov, Zh. Eksp. Teor. Fiz. 39, 1781 (1960)[Sov. Phys. JETP 12, 1243 (1961)].

[6] A. M. Clogston, Phys. Rev. Lett. 9, 266 (1962).

[7] B. S. Chandrasekhar, Appl. Phys. Lett. 1, 7 (1962).

[8] P. Fulde and R. A. Ferrell, Phys. Rev. 135, A550 (1964).

[9] A. I. Larkin and Yu. N. Ovchinnikov, Zh. Eksp. Teor. Fiz. 47, 1136 (1964)[Sov. Phys. JETP 20, 762 (1965)].

[10] K. Blagoev, J. Engelbrecht, and K. Bedell, Phys. Rev. Lett. 82, 133 (1999).

[11] H. Suhl, Phys. Rev. Lett. 87, 167007 (2001).

[12] A. Abrikosov, J. Phys. Cond. Matt. 13, L943 (2001).

[13] M. Cuoco, P. Gentile, and C. Noce, Phys. Rev. B 68, 054521 (2003).

[14] T. R. Kirkpatrick, D. Belitz, T. Vojta, and R. Narayanan, Phys. Rev. Lett. 87, 127003 (2001).

[15] K. Machida and T. Ohmi, Phys. Rev. Lett. 86, 850 (2001).

[16] M. B. Walker and K. V. Samokhin, Phys. Rev. Lett. 88, 207001 (2002).

[17] N. I. Karchev, K. B. Blagoev, K. S. Bedell, and P. B. Littlewood, Phys. Rev. Lett. 86, 846 (2001).

[18] R. Shen, Z. M. Zheng, S. Liu, and D. Y. Xing, Phys. Rev. B 67, 024514 (2003).

[19] J. E. Hirsch, Phys. Rev. B 40, 2354 (1989);40, 9061 (1989);59, 6256 (1999). 